\title{
Viviendas sociales de la Ciudad de Santa Fe. Cómo mejorar su Etiqueta de Eficiencia Energética. Caso de estudio: Vivienda Universal
}

\section{Social housing of the City of Santa Fe. How to improve your Energy Efficiency Label. Case study: Universal Housing}

Sebastián Estanislao Puig, Romina Sol Alberini, Agustina Eggel

Laboratorio de Técnicas y Materiales (LATMAT) de la Facultad de Arquitectura, Diseño y Urbanismo de la Universidad Nacional del Litoral, Santa Fe, Argentina

spuig@fadu.unl.edu.ar

\section{RESUMEN}

El siguiente trabajo tiene como objetivo evaluar la incidencia de mejoras tecnológicas, en relación a la eficiencia energética de las envolventes en viviendas sociales. Esto se realiza a través de la aplicación del Índice de Prestaciones Energéticas (I.P.E), definido por el Programa Nacional de Etiquetado de Viviendas basándose en la Norma IRAM 11900. El caso de estudio es la denominada "Solución habitacional de dos dormitorios o Vivienda Universal (VU) Posición 2", desarrollada por la Dirección Provincial de Vivienda y Urbanismo de la provincia de Santa Fe, construida en el barrio Coronel Dorrego de la Ciudad de Santa Fe. Se busca comparar el I.P.E actual del prototipo, con el obtenido luego de la intervención tecnológica. Como resultado, el prototipo alcanza un I.P.E de $592 \mathrm{kwh} / \mathrm{m}^{2}$ año. Al incorporar variantes de mejoras en forma progresiva, se logran mejoras del $78 \%$, lo cual representa un importante ahorro energético.

\section{ABSTRACT}

The following work aims to evaluate the incidence of technological improvements, in relation to the energy efficiency of the envelopes in social housing. This is done through the application of the Energy Performance Index (IPE), defined by the National Housing Labeling Program based on the IRAM 11900 Standard. The case study is the so-called "Two-bedroom housing solution or Universal Housing (VU) Position 2", developed by the Provincial Directorate of Housing and Urbanism of the province of Santa Fe, built in the Coronel Dorrego neighborhood of the City of Santa Fe. It seeks to compare the current IPE of the prototype, with that obtained after the technological intervention. As a result, the prototype achieves an I.P.E of $592 \mathrm{kwh} / \mathrm{m}^{2}$ year. By incorporating variants of improvements progressively, improvements of $78 \%$ are achieved, which represents significant energy savings.

PALABRAS CLAVES: Indice de prestaciones Energéticas-IPE, Solución habitacional, ahorro energético, prototipo, intervención tecnológica.

KEY WORDS: Energy performance index-IPE, Housing solution, energy saving, prototype, technological intervention.

FECHA DE RECEPCIÓN: 13/9/2021| FECHA DE ACEPTACIÓN: 27/10/2021

DOI: http://dx.doi.org/10.30972/arq.0185675 


\section{INTRODUCCIÓN}

En 2013, el Grupo Intergubernamental de Expertos sobre el Cambio Climático (IPCC), creado por la Organización Meteorológica Mundial (OMM) y la ONU Medio Ambiente, proporcionó claridad sobre el papel de la actividad humana en el cambio climático cuando publicó su Quinto Informe de Evaluación. Su conclusión fue, que el cambio climático es real y las actividades humanas son sus principales causantes.

Los impactos de este cambio climático, que ya quedaban en evidencia en las conclusiones que se producían en la Convención Marco de las Naciones Unidas sobre el Cambio Climático (CMNUCC), son: la disminución o pérdida de glaciares, el aumento paulatino de la temperatura promedio a nivel regional y mundial, transformaciones en los regímenes de precipitaciones, pérdida de biodiversidad, entre otras.

La principal causa de este cambio climático son las emisiones de dióxido de carbono $\left(\mathrm{CO}_{2}\right)$ nivel global que aumentan la temperatura planetaria y el nivel de las aguas, lo que se refleja en la frecuencia de inundaciones, problema recurrente y que constituye una de las principales causas de desastres en la Ciudad de Santa Fe, Argentina.

Según el Programa de las Naciones Unidas para el Medio Ambiente (PNUMA), las emisiones de $\mathrm{CO}_{2}$ fruto de mantener operativos edificios aumentaron en 9,95 gigatoneladas en 2019, lo que junto a la contaminación generada por la industria de la construcción aglutina el $38 \%$ de las emisiones globales de $\mathrm{CO}_{2}$ relacionadas con la energía. Por lo tanto, es importante que desde la disciplina, podamos investigar y brindar herramientas que aporten al medio ambiente, apuntando a una mejora en las condiciones de confort y salud de sus ocupantes, brindando una respuesta sustentable y a largo plazo.

El siguiente trabajo se enmarca en una Tesis de Grado de la Facultad de Arquitectura, Diseño y Urbanismo (FADU) de la Universidad Nacional del Litoral (UNL) con el nombre "Viviendas sociales de la Ciudad de Santa Fe. Cómo mejorar su categoría de Etiquetado de Eficiencia Energética a partir de estrategias pasivas y sistemas activos" con el objetivo de proponer mejoras que aumenten la eficiencia energética y el confort de las viviendas sociales proyectadas por el Estado para la Ciudad de Santa Fe.

\section{METODOLOGÍA}

El trabajo se basa en el análisis del I.P.E de la norma IRAM 11900/17, a través de la utilización del aplicativo elaborado por la Secretaria de Energía de estado de la Provincia de Santa Fe que fue cedido a la Secretaria de Energía de la Nación para poder aplicarlo en las provincias que firmaron los correspondientes convenios.

Se establece el "Indice de Prestación Energética" de un inmueble (IPE) como la cantidad estimada de energía primaria que demandaría la normal utilización de un inmueble durante un año y por metro cuadrado satisfaciendo las necesidades asociadas únicamente a calefacción invernal, climatización estival, agua caliente sanitaria e iluminación, según niveles de confort establecidos por las mejores prácticas vigentes y estándares internacionales. Dicho índice será un valor numérico y se medirá en $\mathrm{kwh} / \mathrm{m}^{2}$ año de energía primaria. (Ley Provincial de Santa Fe 13903, 2019).

A esta valoración la autoridad de aplicación establece un modelo de Etiqueta en la que figuran siete (7) categorías nomencladas desde la letra A (mayor nivel de eficiencia energética) hasta la letra $G$ (menor nivel de eficiencia energética), estableciendo además la correspondencia entre cada letra y rangos de valores del IPE.

Para llevar adelante el análisis del prototipo, se utilizo la matriz operativa elaborada por Puig y San Juan (2020), presentada en el paper "Intervención tecnológica en la vivienda social para 
maximizar la eficiencia energetica, aplicando el indice de prestaciones energéticas (I.P.E.). Caso de estudio: Vivienda Universal, Santa Fe, Argentina".

\section{Caso de estudio}

El caso de estudio corresponde a la denominada "Solución habitacional de dos dormitorios o Vivienda Universal de dos dormitorios (V.U.) Posición 2" desarrollada por la Dirección Provincial de Vivienda y Urbanismo (DPVyU) de la Provincia de Santa Fe. (Figuras 3 y 4) se ubica en el barrio Coronel Dorrego de la Ciudad de Santa Fe. (Figura 1 y 2 )
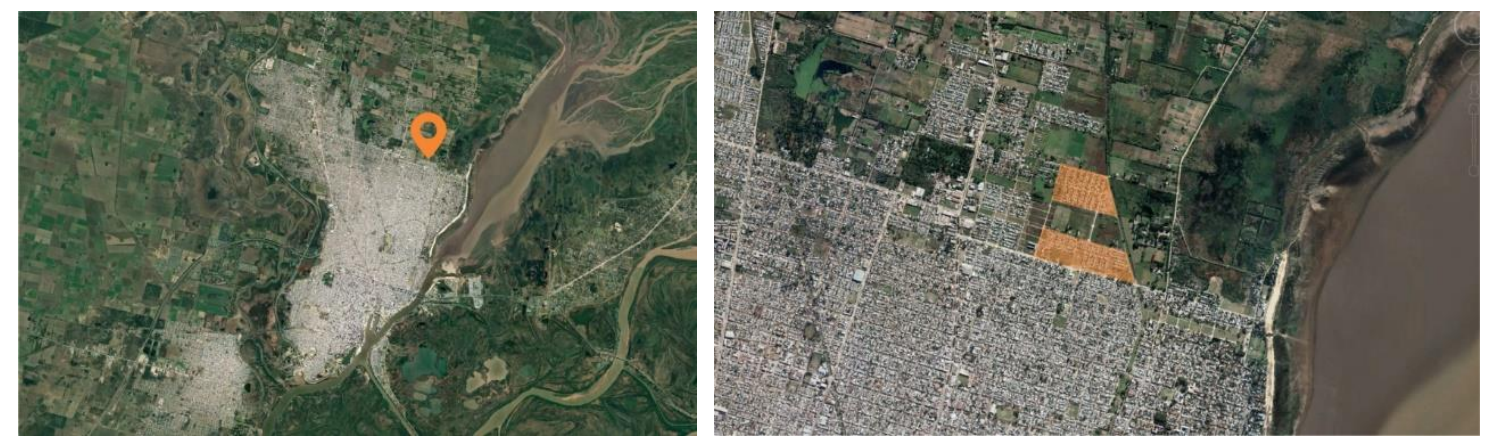

Figura 1 y 2: Localización del barrio "Coronel Dorrego" dentro de la Ciudad de Santa Fe, donde fueron construidas viviendas sociales con el prototipo Vivienda Universal (VU). Fuente: Google Earth.
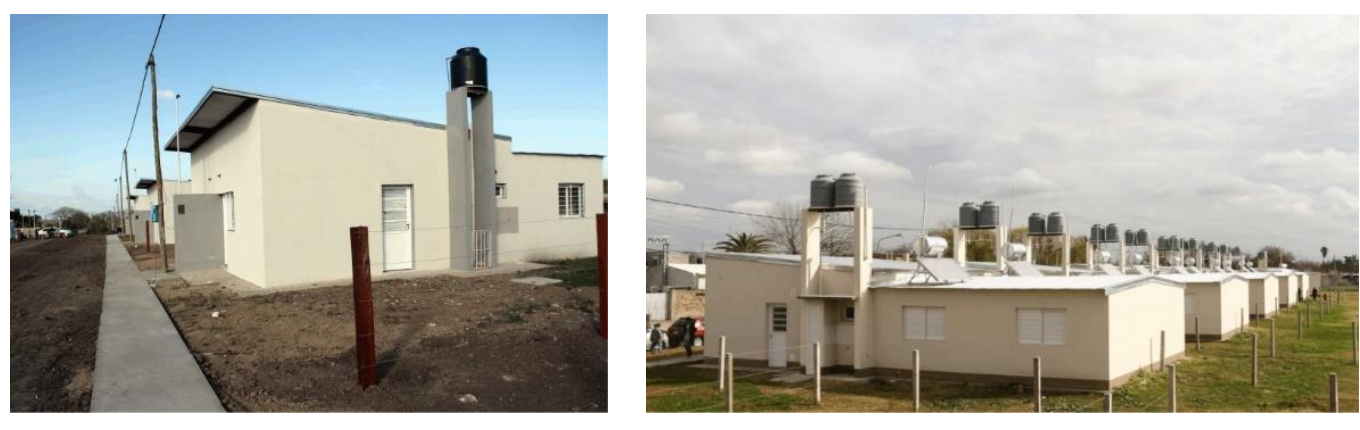

Figuras 3 y 4: Vivienda Universal (VU) Posición 2 - DPVyU. Fuente: Fotos propias del Relevamiento y Diario El litoral.

De acuerdo a la Norma IRAM 11.603 (1996) se localiza en la Zona Bioambiental Cálida (II) de la República Argentina, subzona: Cálida húmeda (Ilb), donde el verano es la estación mas crítica con valores de temperatura media de $24^{\circ} \mathrm{C}$ y máxima superiores a $30^{\circ} \mathrm{C}$. Las mayores amplitudes térmicas se dan en esta época del año, con valores que no superan los $16^{\circ}$. El invierno presenta bajas amplitudes térmicas y temperaturas medias que oscilan entre $8^{\circ} \mathrm{C}$ y $12^{\circ} \mathrm{C}$.

En cuanto a su implantación, se consideró a la vivienda como aislada, excenta de toda edificación lindera, por lo tanto todos sus muros están expuestos al exterior y sin ningún tipo de obstáculo físico. Con respecto a su orientación, su lado más desfavorable, el de mayor cantidad de aberturas, se destinó al Norte, considerado el punto cardinal de mayor ganancia solar.

La V.U. cuenta con $63,08 \mathrm{~m}^{2}$ de superficie cubierta, 2 dormitorios, cocina integrada al comedor, baño y un lavadero planteado en el exterior de la vivienda (Figura 5). El prototipo está materializado por las siguientes soluciones constructivas: muros exteriores e interiores de ladrillos cerámicos huecos portantes, cubierta liviana de chapa galvanizada con aislación térmica y cielorraso suspendido y carpinterías de aluminio con vidrio simple de $3 \mathrm{~mm}$. 
Viviendas sociales de la Ciudad de Santa Fe. Cómo mejorar su Etiqueta de Eficiencia Energética. Caso de estudio: Vivienda Universal

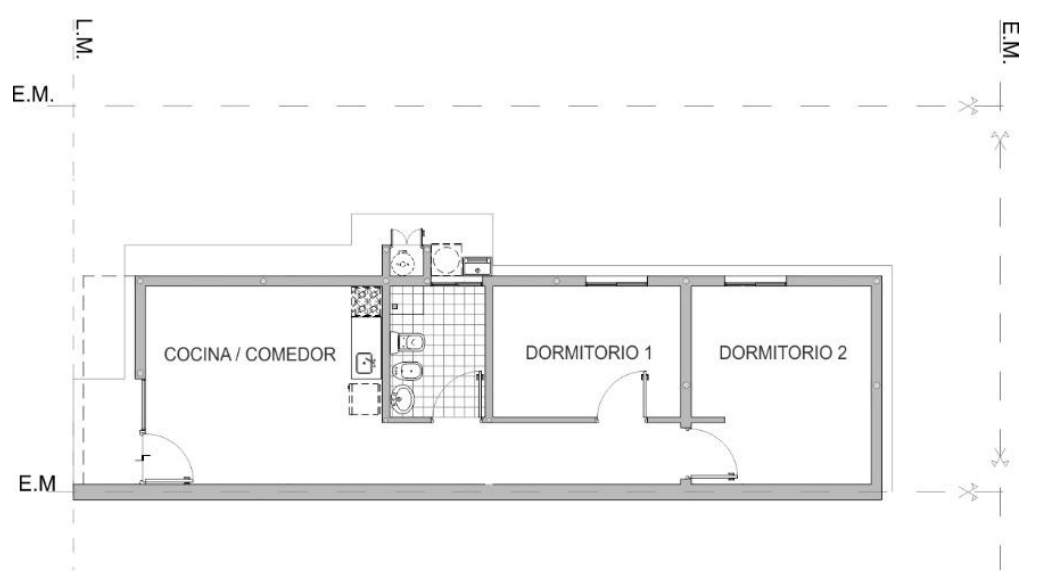

Figura 5: Planta de la Vivienda Universal -VU- Posición 2. DPVyU. Fuente: www.santafe.gov.ar

\section{DESARROLLO}

Análisis del IPE de la Vivienda Universal (VU).

Situación 0. "Caso Base"

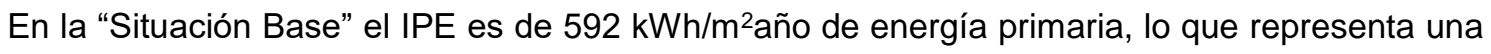
Categoría de Etiqueta de Eficiencia Energética " $G$ ".

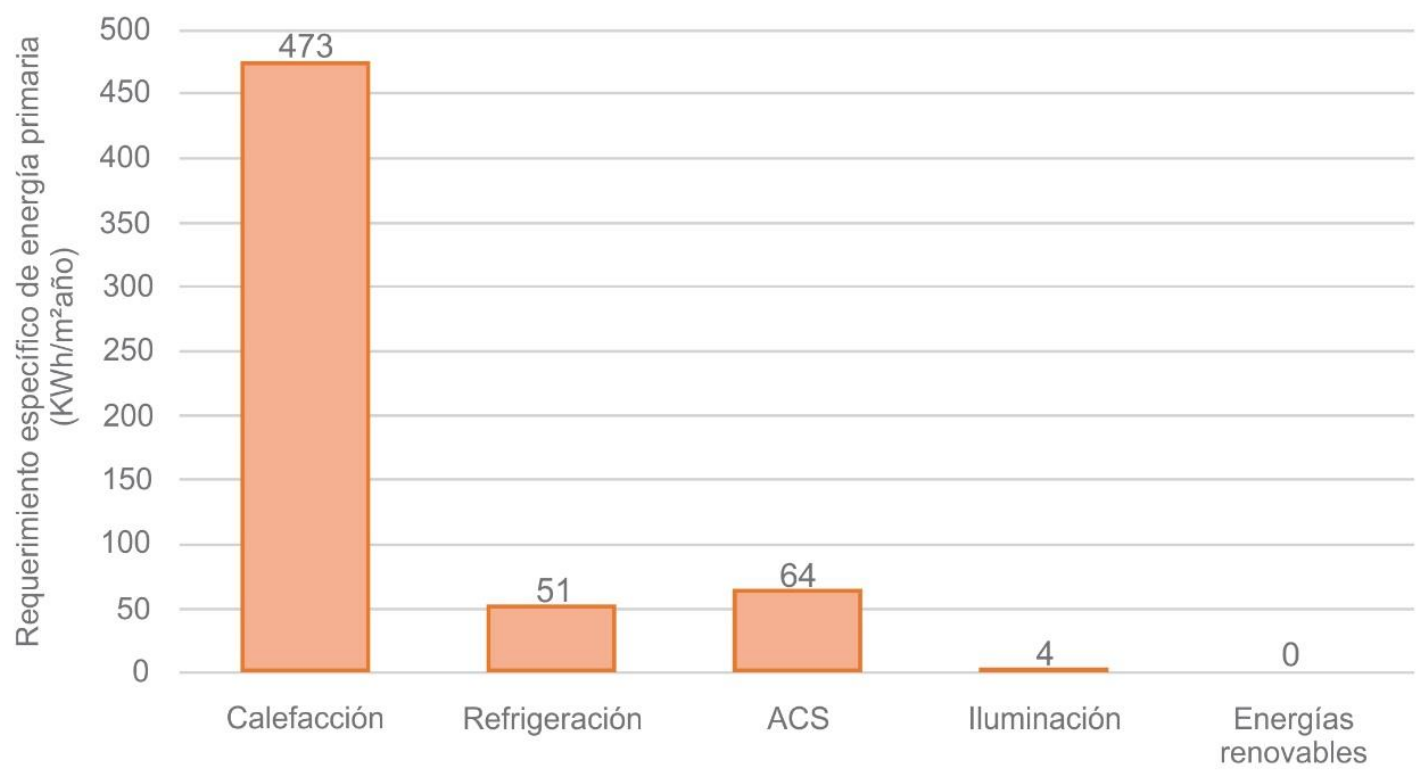

Figura 6: Gráfico de Requerimiento específico de energía primaria. Fuente: Elaboración propia. Aplicativo de Etiquetado de viviendas.

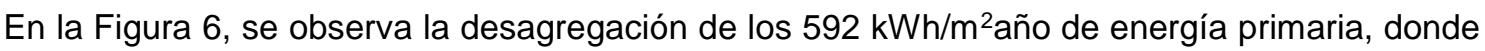
el $79.9 \%$ del requerimiento energético es utilizado para calefaccionar la vivienda en invierno. Este porcentaje tan elavado se debe a que en este tipo de prototipos no contemplan en sus pliegos licitatorios sistemas activos de climatización. Por esta razón es que el aplicativo informático asume que la Zona Térmica (Z.T.) de estudio será acondicionada con los sistemas menos eficientes. A esta situación, se agrega la deficiente resistencia térmica tanto de la cubierta como del resto de las envolventes.

En la Figura 7, se detallan las pérdidas térmicas de todos los elementos de la vivienda en el mes de julio. De un total de $3322,58 \mathrm{~W}$ el $46,65 \%$ son pérdidas de la Cubierta $(1550,06 \mathrm{~W})$, el $37,48 \%$ 
de Muros perimetrales (1245,38 W), el 9,54\% de Aberturas $(317,07 \mathrm{~W})$ y el $6,34 \%$ del Solado $(210,63 \mathrm{~W})$.En general, casi todos los elementos poseen elevados valores en cuanto a sus pérdidas térmicas, sobre todo en el mes de julio, el de mayor incidencia en el balance de pérdidas energéticas para el período de calefacción.

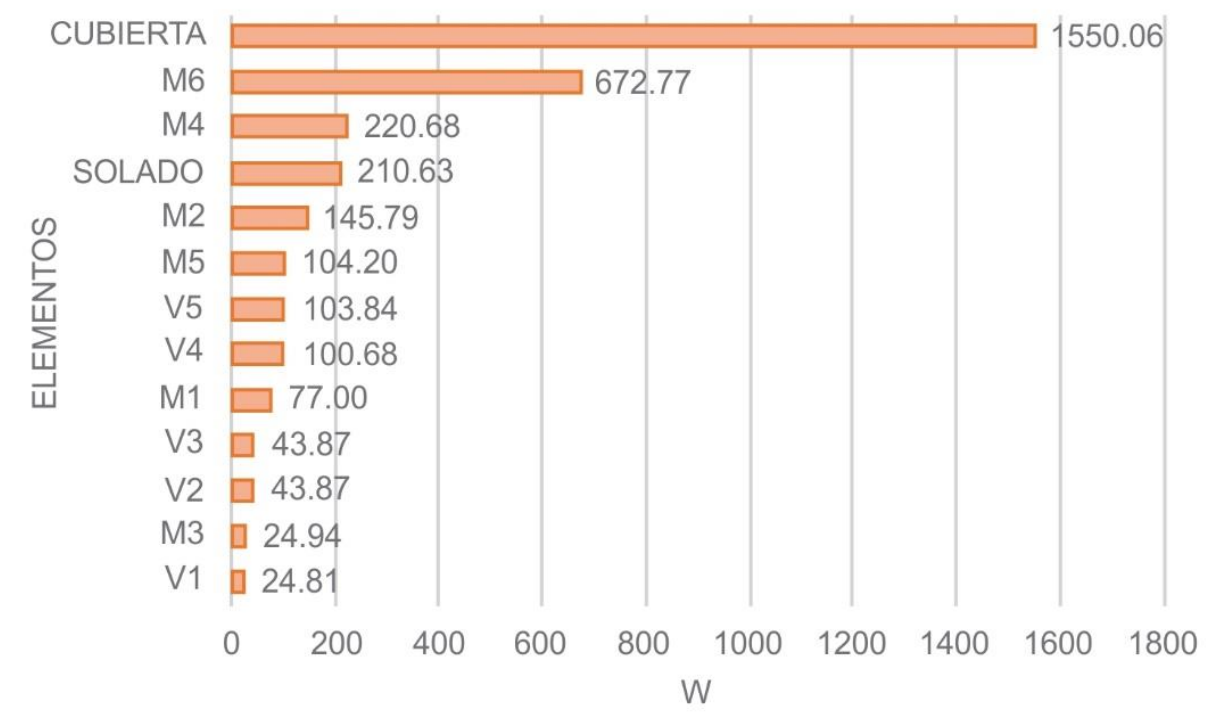

Figura 7: Vivienda Universal -VU- Posición 2. DPVyU. Pérdidas Térmicas de cada elemento expresadas en Watts para el mes de julio. Fuente: Aplicativo de etiquetado de Viviendas. Elaboración Propia.

El Muro Exterior 6 (M6), es el elemento que presenta mayores pérdidas de energía en el prototipo (Figura 8) con 672,77 W. Esto se debe a que es el componente de mayor tamaño en comparación al resto de las envolventes verticales, así como también a que posee una baja resistencia térmica en los componentes del sistema constructivo.

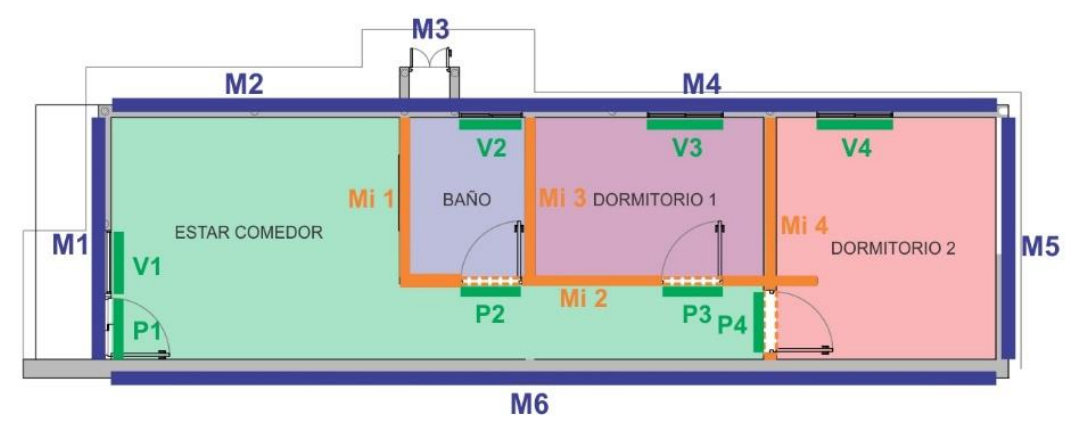

Figura 8: Vivienda Universal -VU- Posición 2. DPVyU. Codificación de los elementos para carga de información en el aplicativo de etiquetado. Fuente: Elaboración Propia

\section{Situación 1: Nivel "B" de IRAM para Cubierta}

Como se mecionó anterioremente el elemento más crítico, que tiene mayores pérdidas térmicas en el mes de julio y mayores ganancias solares en enero, es la cubierta. La solución constructiva actual no verifica ninguno de los niveles de confort de la norma IRAM 11.605: "Acondicionamiento Térmico de Edificios. Condiciones de habitabilidad en Edificios". Se decidió mejorar esta envolvente reemplazando la membrana de espuma de polietileno con aluminio indicada en el Pliego de Especificaciones Técnicas, por un Fieltro de Lana de Vidrio de $80 \mathrm{~mm}$ de espesor con una densidad de $31 \mathrm{~kg} / \mathrm{m}^{3}$. Con este cambio, se obtiene un Nivel "B" de confort en la IRAM 11.605 
y si bien no se sube de categoría en la Etiqueta de Eficiencia Energética se logra una disminución de un $37 \%$ en el I.P.E. de la situación base ( $373 \mathrm{~kW} / \mathrm{m} 2$ año).

\section{Situación 2: Apareamiento de Prototipos}

Como segunda medida, se consideró al prototipo apareado a partir de su Muro Exterior 6 (M6). Esta estrategia, anula las pérdidas y ganancias de este elemento ya que dejará de estar expuesto a agentes externos y pasara a compartir adyacencia a otra Z.T. climatizada. Con ésta mejora, se logra mejorar la categoría de la Etiqueta, pasando de una letra "G" a una Etiqueta "E" con un IPE de $247 \mathrm{~kW} / \mathrm{m}^{2}$ año, lo que representa un incremento en la efectividad de la estrategia de un $58 \%$ con respecto a la situación de base.

Esta acción no tiene costo y es considerada por el proyectista. Se debe de tener siempre en cuenta debido a la incidencia que tiene en la mejora de las prestaciones energéticas de los prototipos.

\section{Situación 3: Nivel "B" de IRAM para Muros Exteriores}

La solución constructiva antes mencionada de envolventes verticales (muros) verfica el Nivel de confort "C" de la norma IRAM 11.605. Para llegar al Nivel "B", se plantea el reemplazo de ladrillos cerámicos huecos de $18 \times 19 \times 33 \mathrm{~cm}$ por Ladrillos de HCCA (Hormigón celular curado en autoclave) de un espesor de $20 \mathrm{~cm}$. Este es un material compuesto por microburbujas de aire incorporadas en su masa, actúan como si fueran millones de pequeñas "cámaras de aire", por este motivo es que posee un coeficiente de conductividad térmica muy bajo respecto a otros materiales de construcción. Elegir este material significa mejoras no sólo a nivel de Eficiencia Energética sino también simplicidad de construcción ya que se adapta a todo tipo de obras nuevas, remodelaciones o ampliaciones.

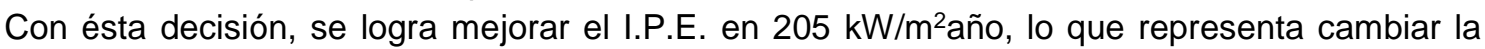
categoría de la Etiqueta, que al momento tiene una letra "E" por una Etiqueta "D". Esta estrategia incrementa la efectividad del prototipo en un $65 \%$ con respecto a la situación de estudio.

\section{Situación 4: Mejora de condición térmica en Solados}

El pliego de especificaciones técnicas no solicita que el solado disponga de aislación. Para mejorar sus prestaciones energéticas es que se se incorpora a la solución constructiva de la Situación Base (Contrapiso + Carpeta) el siguiente material: aislación térmica perimetral bajo contrapiso del lado interior de la vivienda de $50 \mathrm{~cm}$ de ancho compuesto de poliestireno expandido de $2 \mathrm{~cm}$ de espesor y $25 \mathrm{~kg} / \mathrm{m}^{3}$ de densidad. Esta decisión responde a la recomendación efectuada en la Norma IRAM 11604.

De ésta manera, se logra un descenso en el IPE a $194 \mathrm{~kW} / \mathrm{m}^{2}$ año, permaneciendo en la etiqueta "D".

\section{Situación 5: Incorporación de Energía Renovable - Agua Caliente Sanitaria (ACS) Solar Térmica}

A la provisión de Agua Caliente Sanitaria que el prototipo estipula en su pliego licitatorio, se incorpora un sistema de ACS Solar Térmica. El mismo esta compuesto por un equipo que posee un colector solar del tipo de tubos de vacío, con un área de captación de 2,03 $\mathrm{m}^{2}$ y una capacidad de almacenamiento de 150 litros, la inclinación que se utiliza es de $30^{\circ}$ y se orienta el área de capatación hacia el cardinal Norte. El equipo utilizado, tiene un coeficiente global de pérdidas térmicas de 4 y un factor de eficiencia óptica de 0,74 . Con esta medida, se logra llegar a una etiqueta "C" con $132 \mathrm{~kW} / \mathrm{m}^{2} a n ̃ o$, que representa una efectividad en la mejora del prototipo en el rango del $78 \%$. 


\section{DISCUSIÓN DE RESULTADOS}

Del análisis efectuado con el aplicativo de etiquetado de vivienda arroja que el IPE de la Vivienda Universal (VU) Posición 2 es de 592 kWh/m²año. Si la Ley 13.903 "Etiquetado de Eficiencia Energética de Inmuebles destinados a Vivienda" estaría reglamentada, para este caso en particular se le asignaría una etiqueta "G". Con el fin de establecer un objetivo real y factible, se decide llegar a la Clase "C" de la Etiqueta de Eficiencia Energética, ya que el artículo 16 de dicha ley establece que el Estado Provincial implementará estándares mínimos de eficiencia energética en todos los planes de vivienda que sean desarrollados con presupuesto propio. El Poder Ejecutivo fijará dichos estándares mínimos de manera gradual y progresiva comprometiéndose a lograr como mínimo la clase de eficiencia energética $C$ para todas las viviendas que sean ejecutadas a partir del año 2027.

Con las estrategias e implementaciones relacionadas a las mejoras de la envolvente edilicia y la incorporación de energía renovable, particularmente ACS, Solar Térmica, el aplicativo proyecta un IPE de $132 \mathrm{kWh} / \mathrm{m}^{2}$ año de energía primaria. Esto representa una mejora en la etiqueta que ahora sería categoría "C".

\section{CONCLUSIONES}

En la Figura 9 se sintetizan las situaciones que se plantearon de mejora de la envolvente edilicia, los valores obtenidos del IPE con sus respectivas etiquetas y los porcentejes de insidencia en cuanto a reducción de la demanda energética.

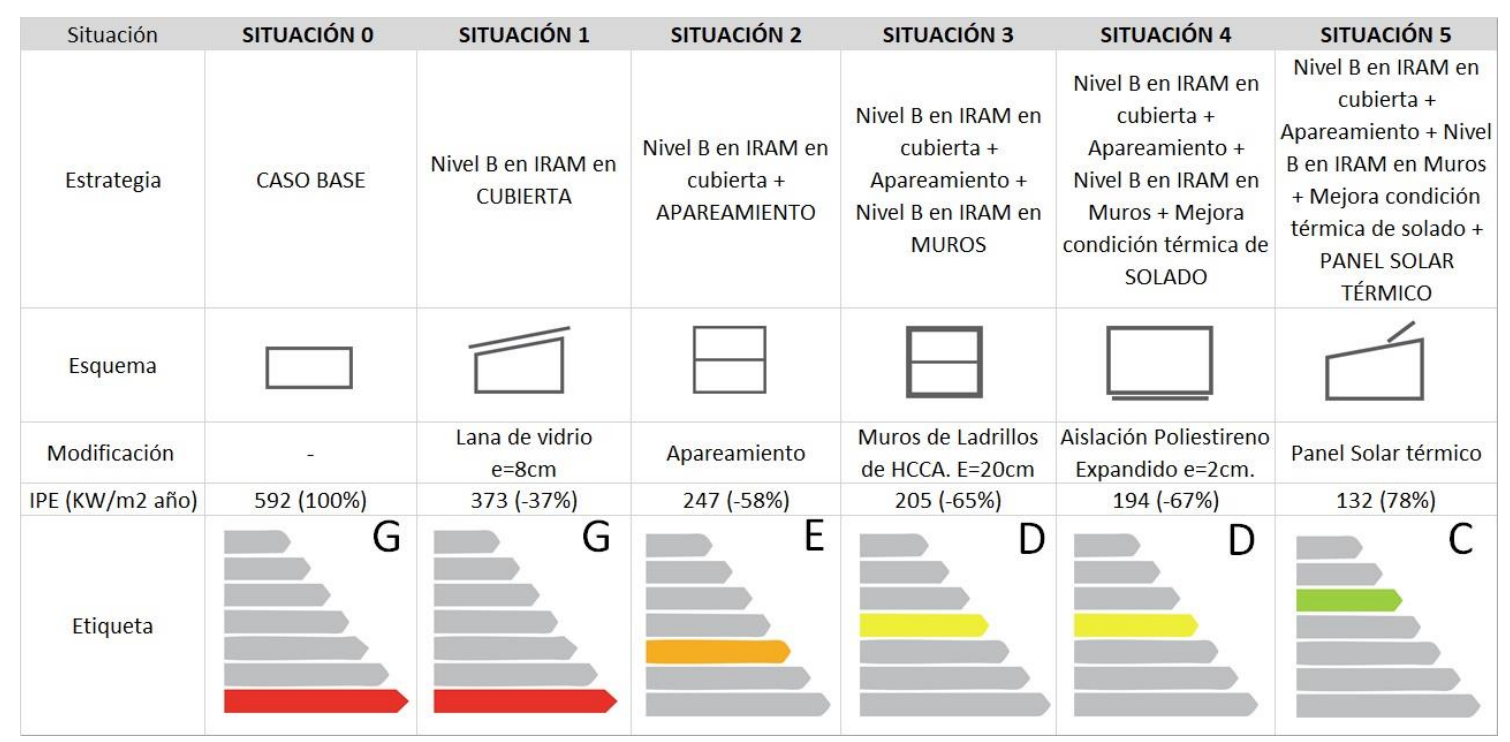

Figura 9: Tabla Resumen de modificaciones realizadas en Vivienda Universal. Fuente: Elaboración propia.

En la situación 0, o también denominada "Caso Base", se observa un valor de IPE de 592

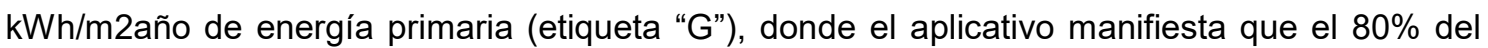
requeremiento energético es utilizado para calefaccionar la $\mathrm{VU}$ en el perído invernal. Esta situación, refleja la utilización de equipamiento ineficiente para acondicionar el espacio habitado y la deficiente resistencia térmica de las envolventes. 
En el análisis particularizado de los componentes de la VU se observó que la Cubierta, es el elemento que posee mayor pérdida de energía. Se plantea la situación 1, optimizando su solución constructiva. De esta forma se logra una eficiencia del 37\% respecto a la Situación Base.

En la situación 2 incorporando la estrategia de diseño de "aparear" dos VU, se logra un valor de IPE de $247 \mathrm{kWh} / \mathrm{m} 2 a n ̃ o$ (Etiqueta E) lo que implica mejorar la performance de la vivienda en un $21 \%$ con respecto a la Situación 1 y un $58 \%$ en relación a la Situación Base.

A partir de esta propuesta es que se ensaya la Situación 3, cambiando la solución constructiva de los muros perimetrales de la VU, se pasa de una Etiqueta "E" a una "D", esto refleja un aumento de su eficiencia en el IPE de un $65 \%$ con respecto a la Situación Base y un $7 \%$ en relación a la Situación 2.

Para la Situación 4, se observa que aislando el solado se obtiene un incremento de efectividad de un $2 \%$ en referencia a la Situación 3. La reducción del IPE a 194 kWh/m2año implica un incremento de efectividad del $67 \%$ en referencia a la situación "Base".

Para la estrategia de incorporación de energías renovables en la Situación 5 se observa una notable reducción en el IPE. Un $11 \%$ Con respecto a la Situación anterior y un $78 \%$ con respecto a la Situación Base, llegando a una Etiqueta de Eficiencia Energética "C" con un IPE de 132 $\mathrm{kWh} / \mathrm{m} 2 a n ̃ o$. Esta situación se debe a que, para el aplicativo de vivienda, la incorporación de energías renovables tiene un impacto directo en la energía primaria que se consume, debido a que la misma se genera directamente en la vivienda y no tiene que ser proveída por sistema energético estatal.

Cabe destacar la importancia de utilizar esta herramienta a nivel Nacional, empezando por el sector público. Pasquevich (2016) sostiene que "(...) es el sector público el que debe tomar la iniciativa y dar el ejemplo a la sociedad y al sector privado". (p. 9). Indica que aplicar el Uso Racional de la Energía Eléctrica (UREE) en la Administración Pública Nacional significaría un efecto multiplicador en el resto del país.

Aislar térmicamente una casa es, sin duda, un beneficio para sus ocupantes, pero ese ahorro energético, si no se propaga a otras casas, barrios y ciudades, es insignificante para el país, casi nulo para el consumo nacional. Por lo contrario, aislar millones de casas, impulsadas por acciones de políticas energéticas y códigos de construcción, posee un enorme efecto en la escala macro. (p. 9)

El Aplicativo Informático Nacional, desarrollado por la Secretaria de Estado de la Energía de la provincia de Santa $\mathrm{Fe}$, es una valiosa herramienta para poder simular la situación actual de una vivienda en su localización. A su vez, los resultados obtenidos demuestran la importancia de incorporar estas herramientas a nivel proyecto, ya que, al realizar un análisis profundo de cada uno de los componentes de la envolvente, nos permite preveer el comportamiento que tendrán en invierno y verano, y de esta forma poder optar entre una u otra tecnología de manera consciente. Asimismo, maximizar la eficiencia energética de las viviendas que diseñamos, disminuyendo su consumo energético y por lo tanto el impacto ambiental.

Lejos de acotar las soluciones constructivas a utilizar en nuestros proyectos, este instrumento nos permite evaluarlas y elegirlas de acuerdo a lo que se quiera lograr tanto a nivel energético como a nivel diseño, ya que no hay una sola soluación energéticamente viable. 


\section{BIBLIOGRAFÍA}

IPCC. International Pannel on Climate Change. (2011). Special Report on Renewable Energy Sources and Climate Change Mitigation. Disponible en: www.ipcc.ch

Ley 13.903 (2019). Cap. I. Disposiciones generales. Art. 4: Índice de Prestación energética. https://www.santafe.gov.ar/normativa/getFile.php?id=1381640\&item $=213203 \&$ cod $=74019$ c0f334b604c35007345bc9a904f

Ley 13.903 (2019). Cap. VII. Viviendas Sociales. Art. 16: Viviendas Sociales.. https://www.santafe.gov.ar/normativa/getFile.php?id=1381640\&item $=213203 \& \operatorname{cod}=74019$ c0f334b604c35007345bc9a904f

Norma IRAM No 11603 (1996). Acondicionamiento térmico de edificios. Clasificación bioambiental de la República Argentina. Instituto Argentino de Normalización y Certificación. Buenos Aires, Argentina.

Norma IRAM N 11604. (2001). Acondicionamiento térmico de edificios. Verificación de sus condiciones higrotérmicas. Ahorro de energía en calefacción. Coeficiente volumétrico $G$ de pérdidas de calor. Cálculo y valores límites. Instituto Argentino de Normalización y Certificación. Buenos Aires, Argentina.

Norma IRAM No 11605. (1996). Acondicionamiento térmico de edificios. Condiciones de habitabilidad en edificios. Valores máximos de transmitancia térmica en cerramientos opacos. Instituto Argentino de Normalización y Certificación. Buenos Aires, Argentina.

Norma IRAM N ${ }^{\circ}$ 11900. (2018). Etiqueta de eficiencia energética de calefacción para edificios. Clasificación según la transmitancia térmica de la envolvente. Instituto Argentino de Normalización y Certificación. Buenos Aires, Argentina.

Pasquevich, D. (2016). Los beneficios del uso racional y eficiente de la energía. En D. M. Pasquevich. (Ed.), Hacia el uso racional y eficiente de la energía en la Administración Pública Nacional. (pp. 25-36). Buenos Aires, Argentina. Editorial. Comisión Nacional de Energía Atómica - CNEA.

Programa Nacional de Etiquetado de Viviendas de Argentina (2019). Presentación y definición del Índice de Prestaciones Energéticas IPE. Disponible en: $<$ https://www.argentina.gob.ar/energia/ahorro-y-eficiencia-energetica/vivienda/programanacional-de-etiquetado-de-viviendas>

Puig, S. y San Juan, G. (2020). Intervención tecnológica en la vivienda social para maximizar la eficiencia energética, aplicando el índice de prestaciones energéticas (IPE). Caso de estudio: vivienda universal, Santa Fe. Argentina. ERMA-Energías Renovables y Medio Ambiente. 1-13. 
